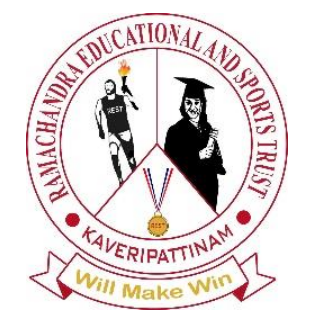

REST Journal on Emerging trends in Modelling and Manufacturing

Vol: 6(2), 2020

REST Publisher

ISSN: 2455-4537

\title{
Website: www.restpublisher.com/journals/jemm
}

\author{
Study on Various Plant Layout used in Flexible Manufacturing System \\ ${ }^{1}$ Jeemit Patel, Karan Patel, ${ }^{2}$ M. Ramachandran \\ ${ }^{1}$ MPSTME, SVKM'S NMIMS University, Shirpur, Dhule, Maharashtra, India \\ ${ }^{2}$ REST Labs, Kaveripattinam, Krishnagiri, Tamil nadu, India
}

\begin{abstract}
With developing interest underway, modern area needs to expand their possibilities underway and adequacy to rival different contenders. For the equivalent, creation measures are to be actualized with lower creation cost and higher adequacy of the business. To tackle this issue, "Plant Layout" comes in picture. Plant format is one of the most significant plan stages in any industry over the world, because of increment in rivalry in measure businesses, climate guidelines and item determination. Plant format for the most part centres around finding and planning of design which lessens the material dealing with cost. Here, we considered a cycle design with rectangular offices utilizing Genetic Algorithm (GA) for its advancement. GA is generally utilized in tackling advancement issues. Because of this, it is considered as a stochastic pursuit calculation having the ability to look internationally. We additionally considered, the idea of reproduction method, which emulates the genuine framework as a model. With this idea we can assess impacts of different procedures in the genuine framework. The Quadratic Assignment Problem (QAP) is planned which appoints each office to one indicated area. For this, a framework-based MILP model was recommended, which can assign everything to at least one than one indicated area. Plant format planned with this procedure brings more noteworthy adaptability, higher viability, and decreased expense underway which is the primary point of any cycle industry.
\end{abstract}

\section{Introduction}

The cycle plant design issue includes choices concerning the spatial allotment of hardware things and the necessary associations among them. Cycle enterprises are compound creation units in which the eventual outcomes are consistently produced through substance and organic cycles or isolating out from crude materials. Designing examinations so as to set the situation requesting and the format of the units and hardware, is one of the principle factors for the cycle plant, assuming a basic function in raising wellbeing levels of these locales, giving monetary conditions with regards to costs and enhancing the usage of these offices. A few distinct methodologies have been considered for the cycle plant format issue. Heuristic methods were recommended with no ensured optimality of the arrangement acquired. In this methodology, we utilize a more practical portrayal of a cycle plant design, considering perspective proportion and direction of cycle units. The info data of any format may change from period to period because of the accompanying variables: Product related: These may remember changes for the plan of an item, in the amount to be created or may include the presentation of another item.

-Product related: These may include changes in the process plan for certain items or may identify with the presentation of new reason apparatus.

- Creation related: Machine breakdowns requiring extensive stretches of personal time, changes in timetables, and inaccessibility of materials or potentially devices might be remembered for this classification.

-Management related: These may remember changes for the association structure that thusly might be brought about by changes in the executives reasoning.

Request related: These may remember occasional changes for request and development or decrease in the interest for an item. They additionally may include uncommon one-time arranges for specific things. The plant design manages the altered plant format to offer the effective work process that gives high efficiency. It is one of the principle fields in mechanical designing where much exploration exertion was spent, and various methodologies were created previously. Format configuration constantly significantly affects the presentation of an assembling or administration industry framework, and subsequently has been a functioning examination zone for quite a few years. A significant part of the plant format plan writing is either algorithmic or of a procedural sort. A few of strategies, for example, Systematic Layout Planning (SLP), Computer program can apply to configuration plant design. The format choice is generally founded on both quantitative and subjective execution appraisals relating to the ideal closeness or closeness connections among the offices. One of the methods is hereditary 
calculation. GA is a hunt and improvement procedure dependent on standards got from nature (i.e., regular determination and hereditary qualities) that is in effect broadly utilized in the arrangement of designing plan issues. GA depend on a nonconcurring ace slave model, as plans may not need a similar measure of calculation exertion and long preparing occasions are relied upon comparative with the correspondence time. The ace cycle will perform GA-related calculations, for example, determination and hybrids. Slave cycles will be mentioned by the ace to finish an investigation of a particular plan and return the processed expense. Customarily, often spent in the plan of a GA has been identified with creating viable wellness capacities and coding plans. The customary way to deal with utilizing hereditary calculations (GAs) to tackle a given issue includes conceiving a method for encoding likely arrangements as strings and utilizing ordinary administrators, ordinarily hybrid and change, to play out the transformative pursuit. Other significant component is "Reproduction" program utilized as logical instruments to help methodically plan the format and to help suitable dynamic to dispense with a portion of the issues. Steps in the relay out and clarification are: a). Examining the current design plant to distinguish the issue on stream of material and activity. b). Planning plant format dependent on SLP way to deal with increment creation limit and diminish material taking care of cost. c). Assessment and choice of choices utilizing Simulation Pro model dependent on two sorts of standards: cost of material taking care of and creation time. Since the reproduced model will show the outcomes and the result of various conditions as presumption in testing phase of the reproduction model. These results help the analyser better comprehend the transient phase of the framework and foresee the impacts that indicated happen during changing the framework. In this work, an overall numerical programming definition for the multi-floor measure plant design issue is introduced. This work broadens the single floor work of aerogun and Rothstein (1998), which depends on a consistent area portrayal. The numerical programming definition limits a cost-based target work subject to hardware direction, non-covering, separation, base and genuine greatest likely harm cost requirements. Ceaseless area MILP models deciding all the while direction and assignment of hardware things have been proposed for the single floor measure plant design issue and reached out to multi floor one by settling on the portion of things to floors through a concurrent, a decay and an iterative methodology. Elective MILP details deciding separations between things by using a piecewise-straight capacity portrayal for outright worth functional were recommended. The accompanying advances were done to advance the design of various offices inside preparing territory - right away, the whole handling zone was discretized into various lattices of fixed size. It was accepted that every unit and office is situated inside these networks. Next, the most basic units of smelling salts plant were distinguished dependent on working temperature, weight and potential results. Four significant offices were additionally chosen for format improvement as for the basic units. The dangers related in every framework were determined utilizing the results examination of each cycle basic unit. Customary breeze plant design improvement has zeroed in on boosting energy creation by putting turbines in the windiest areas while limiting exhibit misfortunes brought about by wake impacts. Notwithstanding, the best in class in-plant configuration has been stretched out to incorporate various targets, for example, generally cost of energy (COE), which considers plant foundation and activities notwithstanding energy creation and even noneconomic goals, for example, commotion alleviation and minimization of ecological effects. The office design issue is frequently defined as a Quadratic Assignment Problem (QAP). The QAP, a very notable model in discrete enhancement, was proposed by Koopmans and Beckmann (1957) unexpectedly to plan the issue of finding offices with material stream between them. As of late, there has been wide enthusiasm for the dynamic adaptation of the issue, which requires managing re-designs after some time, bringing about the Dynamic Plant Layout Problem (DPLP). Thusly, DPLP is an expansion of QAP, described by various periods and a compromise between material taking care of and office moving expenses over an arranging skyline.

\section{Description}

The cycle plant format issue includes choices concerning the spatial distribution of gear things and the necessary associations among them. For the most part, these plant format choices are disregarded or don't get proper consideration during the plan or retrofit of substance plants. When all is said in done handle plant format issue might be described by a few expense or the board/designing drivers, for example, Connectivity cost, development cost, retrofit, wellbeing angles, creation association. [4]. Cycle businesses are synthetic creation units in which the end results are persistently produced through substance and natural cycles or isolating out from crude materials. Designing investigations so as to set the arrangement requesting and the format of the units and hardware, is one of the primary variables for the cycle plant, assuming a crucial function in raising wellbeing levels of these destinations, giving monetary conditions with regards to costs and upgrading the usage of these offices. From a monetary viewpoint, some accept that channelling expenses may incorporate up to $80 \%$ of absolute establishment costs, if there should be an occurrence of improper design of a unit. Likewise, it very well may be presumed that $15 \%$ to $70 \%$ of absolute activity costs in a cycle unit relies upon the method of offices design together. As far as security, the function of episode anticipation is by all accounts substantially huger contrasting with response with mishaps, as practically the entirety of the preparing units manages incredibly perilous materials, for example, poisonous and high potential for fire or blast substances. A legitimate design can forestall event of these occurrences to a huge degree. [7]. The format of 
cycle plant is more mind boggling in that one is attempting to limit various destinations works at the same time, while as yet fulfilling the quantity of limitations. In this methodology, we utilize a more reasonable portrayal of a cycle plant design, considering angle proportion and direction of cycle units. Land cost are planned as being corresponding to the zone of the square shape jumping the units. This more perplexing model of the advancement plant presents troubles to regular numerical programming approaches inferable from the non-differentiability of the goal work. This inspired the utilization of a stochastic advancement procedure, for which there is basically no limitation on the type of the target work. [14]. Various methodologies have been considered for the cycle plant format issue. Heuristic procedures were recommended with no ensured optimality of the arrangement acquired. A diagram parcelling issue was defined to distribute hardware things to segments made by walkways or hallways. A framework based MILP model was recommended, allotting everything to at least one than one competitor areas. Nonstop space MILP models deciding at the same time direction and assignment of gear things have been proposed for the single floor measure plant design issue and reached out to multi floor one by settling on the allotment of things to floors through a concurrent, a decay and an iterative methodology. Elective MILP details deciding separations between things by using a piecewise-direct capacity portrayal for supreme worth functional were recommended. At last, MILP models considering sporadic gear shapes and diverse hardware availability data sources and yields for the single and multi-floor measure plant design issue were introduced. [40]. Plant format has been considered as one of the most significant issues in the plan phase of cycle plants because of the expanding rivalry in measure businesses, limit climate guidelines and item determinations. Here, we centre around the Process Plant Layout issues which help measure engineers fabricate synthetic cycle plants sensibly and monetarily in the plan phase of another plant or during the improvement period of current flowsheets. [49].

\section{Plant Layout}

Plant format is perplexing, in which the land, financing, security and different variables ought to be thought of. The spatial game plan of all cycle units in a given zone ought to amplify the utilization of land and limit the development reserves, costs for material transportation between units, and so on Harmful or dangerous gas for the most part exists in a synthetic plant. The spillage of such perilous gas may prompt harming or blast, for example, BP Texas City occurrence (2005) and Bhopal catastrophe (1984), causing incredible property harm. Thusly, an appropriate format plan should mull over security factors and limit the harm to units and individuals when a surprising occurrence happens. [1]

The plant format manages the altered plant design to offer the effective work process that gives high efficiency. It is one of the fundamental fields in mechanical designing where much exploration exertion was spent, and various methodologies were created previously. Format configuration constantly significantly affects the presentation of an assembling or administration industry framework, and thus has been a functioning examination region for quite a few years. A significant part of the plant format plan writing is either algorithmic or of a procedural sort. A few of strategies, for example, Systematic Layout Planning (SLP), Computer program can apply to configuration plant design. SLP is viewed as one of the elective strategies to configuration plant format. SLP strategy is extremely intriguing on the grounds that it is an essential thing to apply for improving plant format in a wide range of industrial facilities. Along these lines, SLP strategy is investigated as a fundamental instrument to configuration plant format. [3]. The design choice is generally founded on both quantitative and subjective execution evaluations relating to the ideal closeness or closeness connections among the offices. The 'closeness' is an ambiguous thought that catches issues, for example, the material stream and the simplicity of representative management. Obviously, the assessment of basic standards for a format configuration is regularly a difficult and complex assignment. [10]. Planning the new format. The undertaking was started with information assortment on the segments being fabricated at Reynold Gears. Fifteen primary variation models were recognized from PC records. A further examination of the PC records was done to decide the normal week after week request of each model. So as to limit the yearly variety impacts, the information for the normal interest of the past 16 months were utilized. When this was finished, the individual segments of the 15 variation models were removed from the separate. Bill of Materials (BOM). Since Reynold Gears has a working MRPII framework, this was a moderately straightforward undertaking. A sum of 105 sections were recognized which were really machined in the plant (purchased out things which didn't need machining were overlooked). To complete an examination on all the parts would have been dull and wasteful and, thus. 63 parts were chosen utilizing the ABC arrangement (80-20 standard) of need, the need being the recurrence and cost of the segments. [13]. The overall incorporation measure for design assessment incorporates five stages as expressed beneath:

1. Recognize the proper weight, we, to communicate the significance of rule I inside the standard set.

2. Recognize the proper rating, rki, to communicate the reasonableness of elective k regarding standard I.

3. Change the qualities, rki, for both quantitative and subjective lists to a similar scale.

4. Total wi and rki for every choice to get the rating of attractive quality, Dk, for elective $\mathrm{k}$.

5. Select the option with greatest rating esteem, Dk. [19]. The investigation of plant format generally centres around discovering designs that limit the material dealing with cost. The info data required for such issues is the interdepartmental 
material stream framework (IDFM) normally as far as beds, excursions or pounds every day for each period. Here, a period alludes to a timespan during which the info data is expected to stay steady. The info data may change from period to period because of the accompanying elements: Product related: These may remember changes for the plan of an item, in the amount to be created or may include the presentation of another item. Cycle related: These may include changes in the process plan for certain items or may identify with the presentation of new specific reason hardware. Creation related: Machine breakdowns requiring significant stretches of personal time, changes in timetables, and inaccessibility of materials or potentially instruments might be remembered for this class. The executives related: These may remember changes for the association structure that thusly might be brought about by changes in the board reasoning. Request related: These may remember occasional variances for request and development or decrease in the interest for an item. They likewise may include exceptional one-time arranges for specific things. [12]. WITH quick expanding of interest underway, modern processing plants need to build their possibilities underway and adequacy to go up against their rivals. Simultaneously, the creation cycle should be furnished with the capacity to have lower cost with higher adequacy. Hence, the best approach to tackle the issue about the creation is significant. There are numerous ways for example quality control (QC), complete quality administration (TQM), standard time, plant design to take care of the issues concerning profitability. [27]. Target work:

The target work is to limit the absolute expense by considering:

" 1. the upward and even transportation cost;

" 2 . the expense of funnelling;

" 3. the floor development cost (three dimensional issues);

" 4. the land cost (two dimensional issues); [30]. To take care of this issue, a few techniques have been proposed during the most recent twenty years. Strategies dependent on heuristic principles were proposed for the two-dimensional format issues, however there was no assurance that an ideal arrangement would be acquired. A blended number straight programming model (MILP) was created thinking about different sizes and calculations of hardware. Another MILP model was produced for the multi-floor measure utilizing ideas of rectangular shapes and rectilinear separations. However, these strategies have a few constraints since they don't consider security issues. [39]. The next may regularly be viewed as inclinations in design work: 1. gathering hardware of a similar sort 2. organizing hardware as per measure stream. [46]. It might be characterized as a method of finding machines, cycles and plant administrations inside the production line to accomplish the correct amount and nature of yield at the least conceivable expense of assembling. It includes a prudent plan of creation offices so work process is immediate. All things considered, it requires a littler territory or space and can be situated in any sort of working as long as the space is accessible and it is helpful. Plant format for Small Scale business is firmly connected with the processing plant developing and constructed territory. [47].

\section{Genetic Algorithm}

Hereditary calculation (GA) is broadly utilized in taking care of compelled streamlining issues and control methodologies in substance designing. As a result of it, it is a stochastic pursuit calculation with extraordinary globe search capacity, the hereditary calculation is utilized here in managing plant design issue. Another explanation is that the directions of offices can be effectively coded as qualities in GA. [1]. GA is a pursuit and streamlining procedure dependent on standards got from nature (i.e., normal determination and hereditary qualities) that is by and large broadly utilized in the arrangement of designing plan issues. GA depend on an offbeat ace slave model, as plans may not need a similar measure of calculation exertion and long preparing occasions are relied upon comparative with the correspondence time. The ace cycle will perform GA-related calculations, for example, determination and hybrids. Slave cycles will be mentioned by the ace to finish an examination of a particular plan and return the registered expense. Customarily, often spent in the plan of a GA has been identified with creating powerful wellness capacities and coding plans. Rather here, it accentuation on: "the development of populaces of populaces", wherein populaces of applicant arrangements improve their quality through another hybridisation method intended to locate all worldwide optima.; the utilization of a heuristic before the neighbourhood search part of the crossover calculation begins.; the assembly speed of the calculation. [6]. The customary way to deal with utilizing hereditary calculations (GAs) to tackle a given issue includes contriving a method for encoding expected arrangements as strings and utilizing ordinary administrators, commonly hybrid and transformation, to play out the developmental pursuit. The achievement of a GA application, be that as it may, relies upon whether unmistakable portions of the string portrayal speak to legitimate "building blocks" which speak to fractional encodings of good arrangements. [14]. Considered a cycle format including focused rectangular offices and receive a non-differentiable target work, using Genetic Algorithm (GA) for its enhancement. In their methodology security is demonstrated by the Mond fire and wellbeing record which indicates the favoured least separation between measure units. The degree to which these requirements are disregarded decides, despite the fact that not unequivocally, the wellbeing cost of the design. The decision of receiving a GA gets from the natural adaptability that this methodology permits in characterizing and registering a sensible target work (which can be non- 
differentiable), eliminating the imperatives of linearity or the restriction of numerical programming arrangement strategy. [24].

\section{Simulation}

System to relay out the creation floor comprises of three stages, for example, examining the current format, plan the plant design dependent on the SLP and the assessment and determination of elective design utilizing re-enactment program Pro model. Re-enactment program utilized as scientific instruments to help efficiently plan the design and to help proper dynamic to dispense with a portion of the issues. Steps in the relay out and clarification are: a). Breaking down the current format plant to recognize the issue on stream of material and activity. b). Planning plant format dependent on SLP way to deal with increment creation limit and lessen material taking care of cost. c). Assessment and choice of choices utilizing Simulation Pro model dependent on two sorts of standards; cost of material dealing with and creation time. [8]. Idea of re-enactment procedure is to mirror the genuine framework as a model and after that utilization the model to work in numerous conditions and study the impacts to assess the arrangement techniques for the genuine framework. Since the reproduced model will show the outcomes and the symptom of various conditions as presumption in testing phase of the reproduction model. These results help the analyser better comprehend the transient phase of the framework and anticipate the impacts that demonstrated happen during changing the framework. Beginning with plant format planning stage, the physical elective designs were made and contrasted with explore the ideal format in material stream and space prerequisite in each phase of limit development utilizing Pro Model re-enactment running situations. Different targets of the investigation can be summed up as: a). To decide the limit requirements of the creation and the pertinent impact of moving limit limitations in each growing limit stage. b). To amplify the space usage ahead of time considering with the space necessity later on stage. c). To give the rule of limit development between utilizing the options of additional working hours or expanding machines. [26]. In view of the missing of some data from the customary plant format plan, the re-enactment strategy is added to the conventional plant format configuration to show more data, for example, complete time in framework, holding up time, and use. As another idea, there must be a framework that can look for a decent format and afterward can show more significant data about creation. The two ideas are executed as PC programming with the plant format plan module and the plant design recreation module. These modules are created dependent on Microsoft Visual Basic program. To begin with, there is a Microsoft Visual Basic Graphic User Interface (GUI) to get information, for example, item type, item amount, division list, office measurement, From-To Chart. At that point, the plant format plan module is called. This module is additionally evolved dependent on Microsoft Visual Basic program to trade all divisions to get least all out exchange cost between offices. [33].

\section{Optimization}

The accompanying advances were done to improve the format of various offices inside preparing region - right away, the whole handling zone was discretized into various networks of fixed size. It was accepted that every unit and office is situated inside these lattices. Next, the most basic units of smelling salts plant were recognized dependent on working temperature, weight and potential outcomes. Four significant offices were likewise chosen for design advancement regarding the basic units. The dangers related in every lattice were determined utilizing the outcomes examination of each cycle basic unit. Shoot overpressure because of fume cloud blast (VCE) and poisonous delivery outcomes were considered for figuring the related dangers. These danger esteems were changed over into Risk Score utilizing reasonable likelihood capacities. At last, thinking about the monetary boundaries for office establishment and upkeep cost, a numerical definition of all out expense is created. This numerical definition was improved against various imperatives so as to locate the appropriate area of the four chose offices in the alkali cycle plant. [9]. Customary breeze plant design enhancement has zeroed in on boosting energy creation by putting turbines in the windiest areas while limiting exhibit misfortunes brought about by wake impacts. Notwithstanding, the cutting edge in-plant configuration has been reached out to incorporate various goals, for example, generally speaking expense of energy (COE), which considers plant framework and activities notwithstanding energy creation and even noneconomic targets, for example, commotion moderation and minimization of natural effects. For the most part, there is an expanded acknowledgment that coupling over the framework requires more coordinated ways to deal with wind plant plan that think about an enormous scope of choice measures and a more comprehensive way to deal with streamlining. Frameworks designing and multidisciplinary investigation and improvement techniques specifically hold guarantee for improving generally plant plan for a scope of framework goals. A staggered improvement approach was utilized to upgrade the design of the Middle gurned Wind Turbine Cooperative, to amplify wind plant benefit with a general COE objective in which turbine-level controls were an indispensable aspect of the investigation. In any case, the dynamic blend of wind plant controls for expanded energy creation or even diminished turbine loads has not been straightforwardly coupled to wind plant format enhancement. [44].

7.Quadratic Assignment Problem 
The QAP, a very notable model in discrete enhancement, was proposed by Koopmans and Beckmann (1957) unexpectedly to define the issue of finding offices with material stream between them. As of late, there has been wide enthusiasm for the dynamic form of the issue, which requires managing re-formats after some time, bringing about the Dynamic Plant Layout Problem (DPLP). Consequently, DPLP is an augmentation of QAP, described by various periods and a compromise between material taking care of and office moving expenses over an arranging skyline. [6]. The office format issue is frequently defined as a Quadratic Assignment Problem (QAP), which allots m divisions to $\mathrm{m}$ areas while limiting the MHC. Nonetheless, QAP is known to be NP-finished, and advancement techniques are not fit for tackling issues with at least 15 offices in a sensible measure of time. Hence, there is a requirement for heuristic strategies that give great problematic arrangements. [36]. The QAP has been applied to a wide scope of uses, including metropolitan arranging, control board format, and wiring plan. The QAP detailing doles out each division to one area and at most one office to every area. The expense of setting a division at a specific area is subject to the area of the collaborating offices. An ongoing elective definition of the QAP considers relegating interdepartmental separations to division sets. [42]. Koopmans and Beckman (1957) were the first to figure the office format issue as a QAP. The name 'quadratic task issue' was picked in light of the fact that the target work is a seconddegree polynomial capacity of the factors, and the requirements are indistinguishable from the limitations of the task issue. The goal of the QAP is to locate the ideal task of $\mathrm{n}$ offices (plants, offices, or machine stations) to $\mathrm{n}$ destinations so as to limit the material dealing with cost communicated as the result of work process and travel separation. [43].

\section{Mathematical Programming}

In this work, an overall numerical programming definition for the multi-floor measure plant format issue is introduced. This work expands the single floor work of apeirogon and Rothstein (1998), which depends on a persistent area portrayal. [4]. A large portion of the past work on the synthetic plant format utilizing numerical programming methods has been principally cantered around the utilization of uncommon definitions and address an aspect of the design issue. A significant lack is that gear direction isn't thought of. Additionally, cost of land and floor development cost are commonly regularly not considered. Moreover, a considerable lot of the methodologies are just limited to two-dimensional design issues. [30]. The numerical programming plan limits a cost-based target work subject to gear direction, non-covering, separation, base and real most extreme plausible harm cost limitations. [40].

\section{Conclusion}

General cycle plant design is portrayed by a few administration drivers, for example, cost of availability, cost of development, retrofit, wellbeing viewpoints, creation association, the expense of upward and level transportation, and channelling cost. Additionally, it is reasoned that the method of offices design together is reliant on $15 \%$ to $70 \%$ of the absolute activities cost in a cycle unit. At last, MILP models that consider the unpredictable gear shapes and distinctive hardware network sources of info and yields for the single and multi-floor measure plant design issue were introduced. Here, the essential apparatus to configuration plant format is by utilizing the SLP strategy. The inclination of embracing a GA which is gotten from the natural adaptability which will permit in characterizing and processing a sensible target work, disposing of the requirements of linearity or the constraint of numerical programming arrangement technique. The reproduction method is presently added to the conventional plant format configuration to show more information, for example, complete time in framework, season of pausing, and usage. The definition of numerical programming diminishes a cost-based target work subject to gear direction, non-covering, separation, base, and real greatest plausible harm cost limitations. Generally, point here was to decrease cost, increment profitability and productivity through plant format.

\section{References}

[1].Yuan, X. U., W. A. N. G. Zhenyu, and Z. H. U. Qunxiong. "An improved hybrid genetic algorithm for chemical plant layout optimization with novel non-overlapping and toxic gas dispersion constraints." Chinese Journal of Chemical Engineering 21, no. 4 (2013): 412-419.

[2].Watanapa, Anucha, and Wisitsree Wiyaratn. "Systematic layout planning to assist plant layout: case study pulley factory." In Applied mechanics and materials, vol. 110, pp. 3952-3956. Trans Tech Publications Ltd, 2012.

[3].Lin, Lie Chien, and Gunter P. Sharp. "Quantitative and qualitative indices for the plant layout evaluation problem." European Journal of Operational Research 116, no. 1 (1999): 100-117.

[4].Palekar, Udatta S., Rajan Batta, Robert M. Bosch, and Sharad Elhence. "Modeling uncertainties in plant layout problems." European Journal of Operational Research 63, no. 2 (1992): 347-359.

[5].Khan, M. K., and S. H. Gwee. "Plant layout improvements to a medium volume manufacturing system using systematic techniques to form just-in-time manufacturing cells." Proceedings of the Institution of Mechanical Engineers, Part B: Journal of Engineering Manufacture 211, no. 2 (1997): 109-124. 
Patel. et.al / REST Journal on Emerging trends in Modelling and Manufacturing 6(2) 2020, 54-61

[6].Lin, Lie Chien, and Gunter P. Sharp. "Application of the integrated framework for the plant layout evaluation problem." European Journal of Operational Research 116, no. 1 (1999): 118-138.

[7].Shewale, Pramod P., Manmath S. Shete, and S. M. Sane. "Improvement in plant layout using systematic layout planning (SLP) for increased productivity." International journal of advanced engineering research and studies 1, no. 3 (2012): 259261.

[8].Georgiadis, Michael C., Gordian Schilling, Guillermo E. Rotstein, and Sandro Macchietto. "A general mathematical programming approach for process plant layout." Computers \& chemical engineering 23, no. 7 (1999): 823-840.

[9].Park, Kyungtae, Jamin Koo, Dongil Shin, Chang Jun Lee, and En Sup Yoon. "Optimal multi-floor plant layout with consideration of safety distance based on mathematical programming and modified consequence analysis." Korean Journal of Chemical Engineering 28, no. 4 (2011): 1009-1018.

[10].Suzuki, Atsushi, Tetsuo Fuchino, Masaaki Muraki, and Toyohiko Hayakawa. "An evolutionary method of arranging the plot plan for process plant layout." Journal of chemical engineering of Japan 24, no. 2 (1991): 226-231.

[11].Jain, Abhishek, Rajbir Bhatti, and Harwinder Singh. "Improving employee \& manpower productivity by plant layout improvement." In 2014 Recent Advances in Engineering and Computational Sciences (RAECS), pp. 1-6. IEEE, 2014.

[12].Rahman, Shah Md Toufiqur, Md Tausif Salim, and Sultana Razia Syeda. "Facility layout optimization of an ammonia plant based on risk and economic analysis." Procedia Engineering 90 (2014): 760-765.

[13].Fleming, Paul A., Andrew Ning, Pieter MO Gebraad, and Katherine Dykes. "Wind plant system engineering through optimization of layout and yaw control." Wind Energy 19, no. 2 (2016): 329-344.

[14].Patsiatzis, Dimitrios I., and Lazaros G. Papageorgiou. "Optimal multi-floor process plant layout." Computers \& Chemical Engineering 26, no. 4-5 (2002): 575-583.

[15].Latifi, Seyyed Ebrahim, Emran Mohammadi, and Nima Khakzad. "Process plant layout optimization with uncertainty and considering risk." Computers \& Chemical Engineering 106 (2017): 224-242.

[16].Castell, C. M. L., R. Lakshmanan, J. M. Skilling, and R. Banares-Alcantara. "Optimisation of process plant layout using genetic algorithms." Computers \& chemical engineering 22 (1998): S993-S996.

[17].Patsiatzis, Dimitrios I., G. Knight, and L. G. Papageorgiou. "An MILP approach to safe process plant layout." Chemical Engineering Research and Design 82, no. 5 (2004): 579-586.

[18].Patil chetan Vitthal, Chaudhary Saurabh Sanjay, Bhavna R Sharma, M. Ramachandran, Need of Biomedical Waste Management in Rural Hospitals in India, Int. J. Pharm. Sci. Rev. Res., 35(1), 2015, 175-179.

[19].Xu, Gang, and Lazaros G. Papageorgiou. "Process plant layout using an improvement-type algorithm." Chemical Engineering Research and Design 87, no. 6 (2009): 780-788.

[20].Yuan, X. U., W. A. N. G. Zhenyu, and Z. H. U. Qunxiong. "An improved hybrid genetic algorithm for chemical plant layout optimization with novel non-overlapping and toxic gas dispersion constraints." Chinese Journal of Chemical Engineering 21, no. 4 (2013): 412-419.

[21].Rodriguez, Jose M., F. Chris MacPhee, David J. Bonham, and Virendrakumar C. Bhavsar. "Solving the dynamic plant layout problem using a new hybrid meta-heuristic algorithm." International Journal of High Performance Computing and Networking 4, no. 5-6 (2006): 286-294.

[22].Castell, C. M. L., R. Lakshmanan, J. M. Skilling, and R. Banares-Alcantara. "Optimisation of process plant layout using genetic algorithms." Computers \& chemical engineering 22 (1998): S993-S996.

[23].Caputo, Antonio C., Pacifico M. Pelagagge, Mario Palumbo, and Paolo Salini. "Safety-based process plant layout using genetic algorithm." Journal of Loss Prevention in the Process Industries 34 (2015): 139-150.

[24].Rodriguez, Jose M., F. Chris MacPhee, David J. Bonham, and Virendrakumar C. Bhavsar. "Solving the dynamic plant layout problem using a new hybrid meta-heuristic algorithm." International Journal of High Performance Computing and Networking 4, no. 5-6 (2006): 286-294.

[25].Anupama Rajput, M. Ramachandran, V D Gotmare, P. Raichurkar Recent Bioactive Materials for Development of Ecofriendly Dippers: An Overview Journal of Pharmaceutical Sciences and Research, 9(10): 1844-1848, 2017

[26].Pillai, V. Madhusudanan, Irappa Basappa Hunagund, and Krishna K. Krishnan. "Design of robust layout for dynamic plant layout problems." Computers \& Industrial Engineering 61, no. 3 (2011): 813-823.

[27].Meller, Russell D., and Kai-Yin Gau. "The facility layout problem: recent and emerging trends and perspectives." Journal of manufacturing systems 15, no. 5 (1996): 351-366.

[28].Chiang, Wen-Chyuan, and Chi Chiang. "Intelligent local search strategies for solving facility layout problems with the quadratic assignment problem formulation." European Journal of Operational Research 106, no. 2-3 (1998): 457-488.

[29].Suhardini, D., W. Septiani, and S. Fauziah. "Design and simulation plant layout using systematic layout planning." In IOP Conference Series: Materials Science and Engineering, vol. 277, no. 1, p. 012051. IOP Publishing, 2017.

[30].Tearwattanarattikal, Pochamarn, Suwadee Namphacharoen, and Chonthicha Chamrasporn. "Using ProModel as a simulation tools to assist plant layout design and planning: Case study plastic packaging factory." Songklanakarin Journal of Science \& Technology 30, no. 1 (2008). 
[31].Smutkupt, Uttapol, and Sakapoj Wimonkasame. "Plant layout design with simulation." In Proceedings of the International MultiConference of Engineers and Computer Scientists, vol. 2, pp. 18-20. 2009.

[32].Patsiatzis, Dimitrios I., and Lazaros G. Papageorgiou. "Optimal multi-floor process plant layout." Computers \& Chemical Engineering 26, no. 4-5 (2002): 575-583.

[33].Georgiadis, Michael C., Gordian Schilling, Guillermo E. Rotstein, and Sandro Macchietto. "A general mathematical programming approach for process plant layout." Computers \& chemical engineering 23, no. 7 (1999): 823-840.

[34].Patsiatzis, Dimitrios I., G. Knight, and L. G. Papageorgiou. "An MILP approach to safe process plant layout." Chemical Engineering Research and Design 82, no. 5 (2004): 579-586.

[35].Jeevan Kittur, M. Ramachandran, Vishal Fegade, U Ragavendran Numerical Investigation of Total Deformation in roller Bearing using Ansys Analysis, International Journal of Mechanical and Production Engineering Research and Development, 2018, August, 51-58. 